\title{
ENTROPY FOR CANONICAL SHIFTS
}

\author{
MARIE CHODA
}

\begin{abstract}
For a *-endomorphism $\sigma$ of an injective finite von Neumann algebra $A$, we investigate the relations among the entropy $H(\sigma)$ for $\sigma$, the relative entropy $H(A \mid \sigma(A))$ of $\sigma(A)$ for $A$, the generalized index $\lambda(A, \sigma(A))$, and the index for subfactors. As an application, we have the following relations for the canonical shift $\Gamma$ for the inclusion $N \subset M$ of type $\mathrm{II}_{1}$ factors with the finite index $[M: N]$,

$$
H(A \mid \Gamma(A)) \leq 2 H(\Gamma) \leq \log \lambda(A, \Gamma(A))^{-1}=2 \log [M: N],
$$

where $A$ is the von Neumann algebra generated by the two of the relative commutants of $M$. In the case of that $N \subset M$ has finite depth, then all of them coincide.
\end{abstract}

\section{INTRODUCTION}

The notion of the entropy for ${ }^{*}$-automorphisms of finite von Neumann algebras is introduced by Connes and Størmer [3]. In the previous paper [2], we defined the entropy for ${ }^{*}$-endomorphisms of finite von Neumann algebras as an extended version of it. It is possible to define the entropy for a general completely positive linear map $\alpha$ using results in [4] by a similar method. However, the formula of the definition of the entropy for $\alpha$ implies that the entropy is apt to be zero if $\alpha^{k}$ converges to $\alpha$ when $k$ tends to infinity. A conditional expectation is a typical example of such a map. For that reason, interesting completely positive maps $\alpha$ for us to discuss the entropy are those which have the property that $\alpha^{k}$ goes away from $\alpha$ as $k$ tends to infinity.

In this paper, we shall study such a class of ${ }^{*}$-endomorphisms of injective finite von Neumann algebras.

In $\S 3$, we introduce, for a ${ }^{*}$-endomorphism $\sigma$ of an injective finite von Neumann algebra $A$, the notion of an $n$-shift on the tower $\left(A_{j}\right)_{j}$ of finite dimensional von Neumann subalgebras of $A$ which generates $A$ and we obtain the formula of the entropy $H(\sigma)$ for an $n$-shift $\sigma$.

In the work [9] on the classification for subfactors of the hyperfinite type $\mathrm{II}_{1}$ factor, Ocneanu introduced a special kind of *-endomorphism which is called the canonical shift on the tower of relative commutants. The *-endomorphism $\Gamma$ is a generalization of the comultiplication for Hopf algebras and is also considered the canonical shift on string algebras. The *-endomorphism $\Gamma$ has similar properties to the canonical endomorphism of an inclusion of infinite von Neumann algebras due to Longo [7, 8].

Received by the editors October 30, 1989 and, in revised form, September 7, 1990.

1980 Mathematics Subject Classification (1985 Revision). Primary 46L55; Secondary 46L35. 
The canonical shift $\Gamma$ naturally induces a 2 -shift for the injective finite von Neumann algebra $A$ generated by the tower $\left(A_{j}\right)_{j}$ of relative commutants and the entropy $H(\Gamma)$ is determined by the following

$$
H(\Gamma)=\lim _{k \rightarrow \infty} \frac{H\left(A_{2 k}\right)}{k} .
$$

For a *-endomorphism $\sigma$ of a von Neumann algebra $A$, the entropy $H(\sigma)$ is a conjugacy invariant, that is, if there is an isomorphism $\theta$ of $A$ onto a von Neumann algebra $B$ such that $\theta \sigma=\phi \theta$ for a *-endomorphism $\phi$ of $B$, then $H(\sigma)=H(\phi)$. On the other hand, two conjugate *-endomorphisms $\sigma$ and $\phi$ of $A$ give two conjugate von Neumann subalgebras $\sigma(A)$ and $\phi(A)$ under automorphisms of $A$.

In [10], Pimsner and Popa introduced two conjugacy invariants for von Neumann subalgebras. One is the relative entropy $H(A \mid B)$ for a von Neumann subalgebra $B$ of a finite von Neumann algebra $A$, which is defined as an extended version of one for finite dimensional algebras due to Connes-Størmer [3]. The other is the generalized index $\lambda(A, B)$, which plays a role like the index for subfactors due to Jones [6]. In fact in the case of factors $B \subset A$, $\lambda(A, B)^{-1}$ is Jones index $[A: B]$. We shall investigate relations among these invariants.

In $\S 4$, we restrict our attention to finite dimensional von Neumann algebras. We need these results later. The Jones index for a subfactor $N$ of a finite factor $M$ is given as $1 / \tau(e)$ for the projection $e$ of $L^{2}(M)$ onto $L^{2}(N)$ where $\tau$ is the trace on the basic extension algebra of $N \subset M$. In the case of finite dimensional von Neumann algebras, we shall show that the generalized index $\lambda(,)^{-1}$ coincides with Jones index in such a sense.

In $\S 5$, we show that in general the following relation holds for an $n$-shift $\sigma$,

$$
H(A \mid \sigma(A)) \leq 2 H(\sigma) .
$$

A condition under which the equality holds is also given.

In $\S 6$, we obtain the relation between $H(\sigma)$ and $\lambda(A, \sigma(A))$, the generalized index. We define a locally standard tower for $\alpha$ for an increasing sequence $\left(A_{j}\right)_{j}$ of finite dimensional von Neumann algebras. The tower $\left(A_{j}\right)_{j}$ of relative commutants for the inclusion of finite factors $N \subset M$ satisfies this condition. If a ${ }^{*}$-endomorphism $\sigma$ of $A$ is an $n$-shift on a locally standard tower for $\alpha$ which generates $A$, then we have the following:

$$
H(A \mid \sigma(A)) \leq 2 H(\sigma) \leq-\log \alpha \leq \log \lambda(A, \sigma(A))^{-1} .
$$

In $\S 7$, we shall apply the above results to the canonical shift $\Gamma$ for the tower of relative commutants. Let $N \subset M$ be type $\mathrm{II}_{1}$-factors with the finite index. Considering the tower $\left(M_{j}\right)_{j}$ of factors obtained by iterating Jones basic construction from $N \subset M$, we obtain the increasing sequence $\left(A_{j}=M^{\prime} \cap M_{j}\right)_{j}$ of finite dimensional von Neumann algebras. The *-endomorphisms $\Gamma$ is defined on the algebra $\bigcup_{j} A_{j}$ as a mapping such that $\Gamma\left(M_{k}^{\prime} \cap M_{j}\right)=M_{k+2}^{\prime} \cap M_{j+2}$ for all $k \leq j$. First, we remark that $\Gamma$ is extended to the trace preserving *-endomorphism of the finite von Neumann algebra $A=\bigcup_{j}\left(A_{j}\right)^{\prime \prime}$. The *endomorphism $\Gamma$ has an ergodic property that

$$
\bigcap_{k} \Gamma^{k}(A)=C 1
$$


and satisfies all the conditions of the definition for a 2-shift, except one. In order for $\Gamma$ to satisfy all the conditions for a 2-shift, some additional requirement is needed, and in such a case the generalized index $\lambda(A, \Gamma(A))$ is determined by $[M: N]$,

$$
\lambda(A, \Gamma(A))^{-1}=2[M: N] .
$$

For example, in the case where $N^{\prime} \cap M=C 1, \Gamma$ is a 2-shift and the following relation holds

$$
H(A \mid \Gamma(A)) \leq 2 H(\Gamma) \leq 2 \log [M: N] .
$$

Furthermore, if the inclusion $N \subset M$ has finite depth [9, 13], then we have

$$
H(M \mid N)=H(\Gamma)=\log [M: N] \text {. }
$$

In $\S 8$, we discuss conditions for a *-endomorphism $\sigma$ of a factor $M$ to be extended to an automorphism $\theta$ of a factor containing $M$ so that $H(\sigma)=$ $H(\theta)$. If the inclusion $N \subset M$ has finite depth, then $\Gamma$ is extended to an ergodic *-automorphism $\Theta$ which satisfies the following:

$$
H(M \mid N)=H(\Theta)=H(\Gamma)=\log [M: N] \text {. }
$$

\section{PRELIMINARIES}

In this section, we shall fix the notation and terminology used in this paper. Throughout this section $M$ will be a finite von Neumann algebra with a fixed normal faithful trace $\tau, \tau(1)=1$. We equip $M$ with the structure of a preHilbert space by $\langle x, y\rangle=\tau\left(x y^{*}\right)$. Let $\|x\|=\tau\left(x^{*} x\right)^{1 / 2}$ and let $L^{2}(M, \tau)$ by the Hilbert space completion of $M$. Then $M$ acts on $L^{2}(M, \tau)$ be the left multiplication. The canonical conjugation on $L^{2}(M, \tau)$ is denoted by $J=J_{M}$. It is the conjugate unitary map induced by the involution ${ }^{*}$ on $M$. For a von Neumann subalgebra $N$ of $M$, let $e_{N}$ be the orthogonal projection of $L^{2}(M, \tau)$ onto $L^{2}(N, \tau)$. Then the restriction $E_{N}$ of $e_{N}$ to $M$ is the faithful normal conditional expectation of $M$ onto $N$.

The letter $\eta$ designates the function on $[0, \infty)$ defined by $\eta(t)=-t \log t$. For each $k$, we let $S_{k}$ be the set of all families $\left(x_{i_{1}, i_{2}, \ldots, i_{k}}\right)_{i_{j} \in \mathbf{N}}$ of positive elements of $M$, zero except for a finite number of indices and satisfying

$$
\sum_{i_{1}, \ldots, i_{j}, \ldots, i_{k}} x_{i_{1}, \ldots, i_{k}}=1 \text {. }
$$

For $x \in S_{k}, j \in 1,2, \ldots, k$ and $i_{j} \in \mathbf{N}$, put

$$
x_{i_{j}}^{j}=\sum_{i_{1}, \ldots, i_{j-1}, i_{j+1}, \ldots, i_{k}} x_{i_{1}, i_{2}, \ldots, i_{k}} .
$$

Let $N_{1}, N_{2}, \ldots, N_{k}$ be finite dimensional von Neumann subalgebras of $M$. Then

$$
H\left(N_{1}, \ldots, N_{k}\right)=\operatorname{Sup}_{x \in S_{k}}\left[\sum_{i_{1}, \ldots, i_{k}} \eta \tau\left(x_{i_{1}, \ldots, i_{k}}\right)-\sum_{j} \sum_{i_{j}} \tau \eta E_{N_{j}}\left(x_{i_{j}}^{j}\right)\right] .
$$

Let $\sigma$ be a $\tau$-preserving ${ }^{*}$-endomorphism of $M$ and $N$ a finite dimensional von Neumann subalgebra of $M$, then

$$
H(N, \sigma)=\lim _{k \rightarrow \infty} \frac{1}{k} H\left(N, \sigma(N), \ldots, \sigma^{k-1}(N)\right)
$$


exists by [2]. The entropy $H(\sigma)$ for $\sigma$ is defined as the supremum of $H(N, \sigma)$ for all finite dimensional subalgebras $N$ of $M$.

If there exists an increasing sequence $\left(N_{j}\right)_{j}$ of finite-dimensional subalgebras which generates $M$, then by [2]

$$
H(\sigma)=\lim _{j \rightarrow \infty} H\left(N_{j}, \sigma\right) .
$$

The relative entropy $H(M \mid N)$ for a von Neumann subalgebra $N$ of $M$ is defined [10] as an extension form of one [3] by

$$
H(M \mid N)=\operatorname{Sup}_{x \in S_{1}} \sum_{i}\left[\tau \eta\left(x_{i}\right)-\tau \eta E_{N}\left(x_{i}\right)\right] .
$$

This $H(M \mid N)$ is a conjugacy invariant for subalgebras of $M$. Another conjugacy invariant $\lambda(M, N)$ is introduced in [10] as a generalization of Jones index by

$$
\lambda(M, N)=\max \left\{\lambda \geq 0 ; E_{N}(x) \geq \lambda x, x \in M_{+}\right\} .
$$

For an inclusion $N \subset M$ of finite von Neumann algebras, the von Neumann algebra on $L^{2}(M, \tau)$ generated by $M$ and $e=e_{N}$ is called the standard basic extension (or basic construction) for $N \subset M$ and denoted by $M_{1}=\langle M, e\rangle$. Then by the properties of $J=J_{M}$ and $e=e_{N}$, we have $M_{1}=\langle M, e\rangle=J N^{\prime} J$ [6]. If $M_{1}$ is finite and if there is a trace $\tau_{1}$ on $M_{1}$ such that $\tau_{1}(x e)=\lambda \tau(x)$ for all $x \in M$, then the trace $\tau_{1}$ is called the $\lambda$-Markov trace for $N \subset M$. If $M \supset N$ are factors and there is the $\lambda$-Markov trace of $M_{1}$ for $N \subset M$, then Jones index $[M: N]=\lambda^{-1}[6]$.

We shall call an increasing sequence $\left(M_{j}\right)_{j \in \mathbf{N}}$ of von Neumann algebras a standard tower (cf. $[5,9,13]$ ) if $M_{j-1} \subset M_{j} \subset M_{j+1}$ is the basic construction obtained from $M_{j-1} \subset M_{j}$ for each $j$.

Let $L$ be a finite factor containing $M$. We shall call $L$ an algebraic basic construction for the factors $N \subset M$ if there is a nonzero projection $e \in M$ satisfying

(i) $e x e=E_{N}(x) e$ for $x \in M$, and

(ii) $L$ is generated by $e$ and $M$ as a von Neumann algebra.

In this case, there is an isomorphism $\phi$ of $M_{1}$ onto $L$ such that $\phi\left(e_{N}\right)=e$ and $\phi(x)=x$ for all $x \in M$ [11].

We shall call such a projection $e$ a basic projection for $N \subset M$ and a decreasing sequence $\left(N_{j}\right)_{j \in N}$ of finite factors a standard tunnel (cf. [5, 9, 13]) if $N_{j-1} \supset N_{j} \supset N_{j+1}$ is an algebraic basic construction for $N_{j} \supset N_{j+1}$ for each $j$.

\section{ENTROPY OF $n$-SHIFT}

In this section, we shall give the definition of $n$-shifts and a formula of the entropy for $n$-shifts. Let $A$ be an injective finite von Neumann algebra with a fixed faithful normal trace $\tau$, with $\tau(1)=1$. Let $\left(A_{j}\right)_{j=1,2, \ldots}$ be an increasing sequence of finite dimensional von Neumann algebras such that $A=$ the weak closure of $\bigcup_{j} A_{j}=\left\{A_{j}: j\right\}^{\prime \prime}$. Assume that $\sigma$ is a $\tau$-preserving *endomorphism of $A$. Then $\sigma$ is an ultra-weakly continuous, one-to-one mapping with $\sigma(1)=1$. 
Definition 1. Let $n$ be a natural number. A $\tau$-preserving ${ }^{*}$-endomorphism $\sigma$ of $A$ is called an $n$-shift on the tower $\left(A_{j}\right)_{j}$ for $A$ if the following conditions are satisfied:

(1) For all $j$ and $m$, the von Neumann algebra $\left\{A_{j}, \sigma\left(A_{j}\right), \ldots, \sigma^{m}\left(A_{j}\right)\right\}^{\prime \prime}$ generated by $\left\{\sigma^{j}\left(A_{j}\right) ; j=0, \ldots, m\right\}$ is contained in $A_{j+n m}$.

(2) There exists a sequence $\left(k_{j}\right)_{j \in \mathbf{N}}$ of integers with the properties

$$
\lim _{j \rightarrow \infty} \frac{n k_{j}-j}{j}=0,
$$

and

$$
x \sigma^{m}(y)=\sigma^{m}(y) x, \quad \tau\left(z \sigma^{l k_{j}}(x)\right)=\tau(z) \tau(x),
$$

for all $l \in \mathbf{N}, x, y \in A_{j}, m \in k_{j} \mathbf{N}$ and $z \in\left\{A_{j}, \sigma^{k_{j}}\left(A_{j}\right), \ldots, \sigma^{(l-1) k_{j}}\left(A_{j}\right)\right\}^{\prime \prime}$.

(3) Let $E_{B}$ be the conditional expectation of $A$ onto a von Neumann subalgebra $B$ of $A$. Then for $j \geq n, E_{A_{j}} E_{\sigma\left(A_{j}\right)}=E_{\sigma\left(A_{j-n}\right)}$.

(4) For each $j$, there exists a $\tau$-preserving *automorphism or antiautomorphism $\beta$ of $A_{n j+n}$ such that $\sigma\left(A_{n j}\right)=\beta\left(A_{n j}\right)$.

Remark 1 . The number $n$ of an $n$-shift depends on the choice of the sequence $\left(A_{j}\right)_{j}$. Every given $n$-shift can be 1-shift on a suitable tower for the same von Neumann algebra.

Example 1. Let $S$ be the *-endomorphism corresponding to the translation by 1 in the infinite tensor product $R=\bigotimes_{i \in \mathbf{N}}\left(M_{i}, \operatorname{tr}_{i}\right)$ of the algebra $M_{i}$ of $m \times m$ matrices with the normalized trace $\operatorname{tr}_{i}$ on $M_{i}$ for each $i \in \mathbf{N}$. For each $j$, let $A_{j}=\bigotimes_{i=1}^{j}\left(M_{i}, \operatorname{tr}_{i}\right)$. Then for all $n, S^{n}$ is an $n$-shift on the tower $\left(A_{j}\right)_{j}$ for $R$.

In fact, for an $n \in \mathbf{N}$, let $k_{j}=\left[\frac{j}{n}\right]+1$. Then $\left(k_{j}\right)_{j}$ satisfies the following properties $\left(2^{\prime}\right)$ which are stronger than $(2)$ :

$$
\lim _{j \rightarrow \infty} \frac{n k_{j}-j}{j}=0,
$$

and

$$
x \sigma^{m}(y)=\sigma^{m}(y) x, \quad \tau\left(z \sigma^{l k}(x)\right)=\tau(z) \tau(x),
$$

for all $l \in \mathbf{N}, x, y \in A_{j}, k_{j} \leq k, m \in k \mathbf{N}$ and

$$
z \in\left\{A_{j}, \sigma^{k}\left(A_{j}\right), \ldots, \sigma^{k(l-1)}\left(A_{j}\right)\right\}^{\prime \prime} .
$$

It is obvious that other conditions are satisfied by $S^{n}$.

Example 2. Let $\left(e_{j}\right)_{j}$ be the sequence of projections with the following properties for some natural number $k$ and $\lambda \in\left(0, \frac{1}{4}\right] \cup\left\{\frac{1}{4} \cos ^{2}(\pi / n) ; n \geq 3\right\}$,

(a) $e_{i} e_{j} e_{i}=\lambda e_{i}$ if $|i-j|=k$,

(b) $e_{i} e_{j}=e_{j} e_{i}$ if $|i-j| \neq k$,

(c) $\left(e_{j}\right)_{j}$ generates the hyperfinite type $\mathrm{II}_{1}$-factor $R$,

(d) $\tau\left(w e_{i}\right)=\lambda \tau(w)$ for the trace $\tau$ of $R$ and a reduced word $w$ on $\left\{1, e_{1}, \ldots, e_{i-1}\right\}$.

Let $A_{j}$ be the von Neumann algebra generated by $\left\{e_{1}, \ldots, e_{j}\right\}$. Then, by [6], $A_{j}$ is finite dimensional. Let $\sigma$ be the *-endomorphism of $R$ such that $\sigma\left(e_{i}\right)=e_{i+1}$ [1]. Then $\sigma^{n}$ is an $n$-shift on the tower $\left(A_{j}\right)_{j}$ of $R$ for all $n$. In fact, for an $n \in N$, let $k_{j}=\left[\frac{j+k}{n}\right]+1$. Then $\left(k_{j}\right)_{j}$ satisfies properties $\left(2^{\prime}\right)$ in 
Example 1. The conditions (3) and (4) are satisfied by using results in [6 and 1].

In $\S 7$, we shall show that the canonical shift due to Ocneanu is a 2-shift on the tower of relative commutant algebras.

Theorem 1. If a $\tau$-preserving *-endomorphism $\sigma$ of $A$ satisfies the condition (1) and (2) in Definition 1 for the tower $\left(A_{j}\right)_{j}$ of $A$, then

$$
H(\sigma)=\lim _{k \rightarrow \infty} \frac{H\left(A_{n k}\right)}{k} .
$$

Proof. Theorem 1 is a reformulation of Theorem 9 in [2]. We shall repeat a proof of it for the sake of completeness. Since $A$ is approximately finite dimensional, we have by [2]

$$
H(\sigma)=\lim _{j \rightarrow \infty} \lim _{k \rightarrow \infty} \frac{1}{k} H\left(A_{n j}, \sigma\left(A_{n j}\right), \ldots, \sigma^{k-1}\left(A_{n j}\right)\right) .
$$

Hence, by [2 and 3],

$$
\begin{aligned}
H(\sigma) & \leq \lim _{j} \liminf _{k} \frac{1}{k} H\left(\left\{A_{n j}, \ldots, \sigma^{k-j}\left(A_{n j}\right)\right\}^{\prime \prime},\right. \\
& \left.\left.\leq \lim _{j} \liminf _{k} \frac{1}{k}\left[H\left(A_{n j+n(k-j)}\right)+H\left(A_{n j}\right), \ldots, \sigma^{k-1}\left(A_{n j}\right)\right\}^{\prime \prime}\right)\right] \\
& \leq \lim _{j} \liminf _{k} \frac{n k}{k} \frac{\left(A_{n k}\right)}{n k} \\
& =\liminf _{k} \frac{H\left(A_{n k}\right)}{k} .
\end{aligned}
$$

On the other hand, by the condition (2) of $n$-shift,

$$
\frac{1}{k} H\left(A_{j}, \sigma^{k_{j}}\left(A_{j}\right), \ldots, \sigma^{(k-1) k_{j}}\left(A_{j}\right)\right)=H\left(A_{j}\right) \text {. }
$$

Hence by [2 and 3], for a fixed $j$,

$$
\begin{aligned}
k_{j} H(\sigma) & =H\left(\sigma^{k_{j}}\right) \\
& =\lim _{i} \lim _{k} \frac{1}{k} H\left(A_{i}, \sigma^{k_{j}}\left(A_{i}\right), \ldots, \sigma^{k_{j}(k-1)}\left(A_{i}\right)\right) \\
& \geq \lim _{k} \frac{1}{k} H\left(A_{j}, \sigma^{k_{j}}\left(A_{j}\right), \ldots, \sigma^{k_{j}(l-1)}\left(A_{j}\right)\right) \\
& =H\left(A_{j}\right) .
\end{aligned}
$$

This implies that

$$
H(\sigma) \geq \frac{H\left(A_{j}\right)}{k_{j}}=\frac{n}{j} H\left(A_{j}\right)-\frac{H\left(A_{j}\right)}{k_{j}} \frac{n k_{j}-j}{j} .
$$

By the property of $k_{j}$, we have

$$
H(\sigma) \geq \limsup \frac{n}{j} H\left(A_{j}\right) \geq \limsup \frac{H\left(A_{n j}\right)}{j} .
$$

Therefore

$$
H(\sigma)=\lim _{k \rightarrow \infty} \frac{H\left(A_{n k}\right)}{k} .
$$




\section{Finite dimensional algebras}

In this section, $M$ will be a finite dimensional von Neumann algebra and $\tau$ a fixed faithful normal trace of $M$ with $\tau(1)=1$. Then $M$ is decomposed into the direct summands:

$$
M=\sum_{l \in K} \bigoplus M_{l},
$$

where $M_{l}$ is the algebra of $d(l) \times d(l)$ matrices and $K=K_{M}$ is a finite set. Then the vector $d_{M}=d=(d(l))_{l \in K}$ is called the dimension vector of $M$. The column vector $t_{M}=t=(t(l))_{l \in K}$ has $t(l)$ as the value of the trace for the minimal projections in $M_{l}$, and is called the trace vector of $\tau$. Let $N$ be a von Neumann subalgebra of $M$ with $N=\sum_{k \in K_{N}} \oplus N_{k}$. The inclusion matrix $[N \hookrightarrow M]=(m(k, l))_{k \in K_{N}, l \in K_{M}}$ is given by the number $m(k, l)$ of simple components of a simple $M_{l}$ module viewed as an $N_{k}$ module. Then

$$
d_{N}[N \hookrightarrow M]=d_{M} \text { and }[N \hookrightarrow M] t_{M}=t_{N} .
$$

Here we shall give a simple formula for $\lambda(M, N)$.

By the definition of the basic construction of $N \subset M$, there is a natural isomorphism between the centers of $N$ and $\langle M, e\rangle$ via $x \rightarrow J x^{*} J$. Hence there is a natural identification between the sets of simple summands of $N$ and $\langle M, e\rangle$. We put $K=K_{N}=K_{\langle M, e\rangle}$.

The following theorem assures that in the case of finite dimensional von Neumann algebras, the constant $\lambda(\cdot)$ plays the same role as the index for finite factors.

Theorem 2. (1) Assume that there is a trace of $\langle M, e\rangle$ which is an extension of $\tau$. Then

$$
\lambda(\langle M, e\rangle, M)^{-1}=\max _{k \in K} \frac{t_{N}(k)}{t_{\langle M, e\rangle}(k)} .
$$

(2) If the trace $\tau$ of $\langle M, e\rangle$ has the $\tau(e)$-Markov property, then

$$
\lambda(\langle M, e\rangle, M)^{-1}=1 / \tau(e)=\|[N \hookrightarrow M]\|^{2} .
$$

Proof. (1) Let $(a(l, k))_{l \in K_{M}, k \in K_{\langle M, e\rangle}}$ be the inclusion matrix $[M \hookrightarrow\langle M, e\rangle]$. Since $[M \hookrightarrow\langle M, e\rangle]=[N \hookrightarrow M]^{t}[6]$, by the formula in [10],

$$
\lambda(\langle M, e\rangle, M)^{-1}=\max _{k \in K} \sum_{l \in K_{M}} \frac{\min \left\{a(l, k), d_{M}(l)\right\} t_{M}(l)}{t_{\langle M, e\rangle}(k)} .
$$

Since

$$
d_{M}^{t}=\left(d_{N}[N \hookrightarrow M]\right)^{t}=[M \hookrightarrow\langle M, e\rangle] d_{N}^{t},
$$

we have $d_{M}(l)=\sum_{k} a(l, k) d_{N}(k)$. It follows that $d_{M}(l) \geq a(l, k)$ for all $l$ and $k$. Hence

$$
\begin{aligned}
\sum_{l} \min & \left\{a(l, k), d_{M}(l)\right\} t_{M}(l) \\
& =\sum_{l} a(l, k) t_{M}(l)=\left([N \hookrightarrow M] t_{M}\right)(k)=t_{N}(k) .
\end{aligned}
$$

Hence we have

$$
\lambda(\langle M, e\rangle, M)^{-1}=\max _{k \in K} \frac{t_{N}(k)}{t_{\langle M, e\rangle}(k)} .
$$


(2) Let $\lambda=\tau(e)$. Then by [6], the following equivalent statements hold:

$$
\lambda[N \hookrightarrow M][M \hookrightarrow\langle M, e\rangle] t_{N}=t_{N},
$$

and

Hence we have

$$
\lambda[M \hookrightarrow\langle M, e\rangle][N \hookrightarrow M] t_{M}=t_{M} .
$$

$$
t_{N}=[N \hookrightarrow M] t_{M}=[N \hookrightarrow M][M \hookrightarrow\langle M, e\rangle] t_{\langle M, e\rangle}=\frac{1}{\lambda} t_{\langle M, e\rangle} .
$$

Since $1 / \lambda$ is the Perron-Frobenius proper value of $[N \hookrightarrow M][N \hookrightarrow M]^{t}$, we have

$$
\lambda(\langle M, e\rangle, M)^{-1}=\max _{k \in K} \frac{t_{N}(k)}{t_{\langle M, e\rangle}(k)}=\frac{1}{\lambda}=\frac{1}{\tau(e)}=\|[N \hookrightarrow M]\|^{2} .
$$

Definition 2. Let $N \subset M \subset L$ be an inclusion of finite dimensional von Neumann algebras. Then $L$ is said to be an algebraic basic construction for $N \subset M$ if there is a projection $e$ in $L$ satisfying

(a) $L$ is generated by $M$ and $e$,

(b) $x e=e x$ for an $x \in N$,

(c) If $x \in N$ satisfies $x e=0$, then $x=0$,

(d) $e x e=E_{N}(x) e$ for all $x \in M$, ((d) implies (b)).

In this case, there is a ${ }^{*}$-isomorphism of the basic construction $M_{1}=J N^{\prime} J$ onto $L$.

We shall call $N \subset M \subset L$ a locally algebraic extension of $N \subset M$ if there is a projection $p \in L \cap L^{\prime}$ which satisfies that the inclusion $M \subset L p$ is an algebraic basic construction $N \subset M$.

If $L \supset M \supset N$ is a locally standard extension of the inclusion $M \supset N$, we can identity the set $K_{N}$ with a subset of $K_{L}$ via the equality $N e=e(L p) e$. Under this identification, we have the following:

Proposition 3. Let $L \supset M \supset N$ be a locally standard extension of $M \supset N$. Then

$$
\lambda(L, M)^{-1} \geq \max _{k \in K_{N}} \min _{l \in K_{L}} \frac{t_{N}(k)}{t_{L}(l)} .
$$

Proof. Let $(a(k, l))_{k \in K_{M}, l \in K_{L}}=[M \hookrightarrow L]$. Then by [10],

$$
\lambda(L, M)^{-1} \geq \frac{1}{\max _{l} t_{L}(l)} \max _{l} \sum_{k} \min \left\{a(k, l), d_{M}(k)\right\} t_{M}(k) .
$$

Since there is a projection $p \in L \cap L^{\prime}$ which satisfies that $L p$ is isomorphic to the basic extension for $N \subset M$, then $[N \hookrightarrow M]^{t}=\left[M \hookrightarrow L_{p}\right]$. Hence we have, by the same method as in the proof of Theorem 2,

$$
\sum_{k} \min \left\{a(k, l), d_{M}(k)\right\} t_{M}(k)=t_{N}(l),
$$

for $l \in K_{N}$, where we consider $K_{N}$ as a subset of $K_{L}$. Thus

Let

$$
\lambda(L, M)^{-1} \geq \frac{\max _{l \in K_{N}} t_{N}(l)}{\max _{l \in K_{L}} t_{L}(l)} .
$$

$$
I(M)=\sum_{l \in K} d(l) t(l) \log \frac{d(l)}{t(l)},
$$

where $K=K_{M}, d=d_{M}$, and $t=t_{M}$. 
Proposition 4. (i) $H(M \mid N) \leq I(M)-I(N)$,

(ii) $H(\langle M, e\rangle \mid M)=I(\langle M, e\rangle)-I(M)$,

(iii) $I(M) \leq 2 H(M)$ and the equality holds if and only if $M$ is a factor.

Proof. The inequality (i) is an immediate consequence of the following formula [10]

$$
H(M \mid N)=I(M)-I(N)+\sum_{k, l} d_{N}(k) m(k, l) t_{M}(l) \log \min \left\{\frac{d_{N}(k)}{m(k, l)}, 1\right\}
$$

where $(m(k, l))_{k, l}=[N \hookrightarrow M]$.

(ii) By the proof of Theorem 2, $d_{M}(l) \geq a(l, k)$ for all $l \in K_{M}$ and $k \in$ $K_{\langle M, e\rangle}$. It follows that $H(\langle M, e\rangle \mid M)=I(\langle M, e\rangle)-I(M)$.

(iii) Since $d(l) t(l) \leq 1$ for all $l \in K$, we have $I(M) \leq 2 H(M)$. The equality holds if and only if $t(l) d(l)=1$, for some $l$ which means that $M$ is factor.

\section{5. $H(\sigma)$ AND $H(A \mid \sigma(A))$}

In this section we investigate a relation between $H(\sigma)$ and $H(A \mid \sigma(A))$ for an $n$-shift $\sigma$ on the tower $\left(A_{j}\right)_{j}$ for a finite von Neumann algebra $A$.

Let $\left(A_{j}\right)_{j}$ be an increasing sequence of finite dimensional von Neumann algebras. Let $A_{j}=\sum_{k \in K_{j}} \bigoplus A_{j}(k)$ be such a decomposition as in $\S 4$, and $d_{j}$ the dimension vector of $A_{j}$. Then we shall say $\left(A_{j}\right)_{j}$ satisfies the bounded growth conditions [2] if the following two conditions are satisfied:

(i) $\sup _{j}\left|\left(K_{j}\right)\right| / j<+\infty$.

(ii) For some $m, A_{j+1}(l)$ contains at most $d_{j}(k) A_{j}(k)$-components for all $j \geq m$ where $\left|\left(K_{j}\right)\right|$ is the cardinal number of $K_{j}$.

For examples, let us consider the two towers which are treated in Examples 1 and 2. Both of them satisfy the bounded growth conditions [2]. We shall discuss another example in $\S 7$.

Theorem 5. Let $\sigma$ be a $\tau$-preserving ${ }^{*}$-endomorphism of an injective finite von Neumann algebra $A$ with a faithful normal trace $\tau, \tau(1)=1$. If $\sigma$ is an $n$-shift on the tower $\left(A_{j}\right)_{j}$ for $A$, then $H(A \mid \sigma(A)) \leq 2 H(\sigma)$.

Furthermore, if the bounded growth conditions are satisfied, for the tower $\left(A_{n j}\right)_{j}$,

$$
H(A \mid \sigma(A))=2 H(\sigma) .
$$

In order to prove Theorem 5, we need the following:

Lemma 6. Let $\sigma$ be the same as in Theorem 5. If $\sigma$ satisfies the conditions (1), (3), and (4) in Definition 1 for $n$, then

$$
H(A \mid \sigma(A))=\lim _{j \rightarrow \infty} H\left(A_{n j+n} \mid A_{n j}\right) .
$$

Proof. By assumptions, the algebra $A_{n j+n}$ contains $\sigma\left(A_{n j}\right)$. Since two conditional expectations of $A_{n j+n}$ onto $A_{n j}$ and $\sigma\left(A_{n j}\right)$ are conjugate by the automorphism or antiautomorphism $\beta$ of $A_{n j+n}$ in the condition (4),

$$
H\left(A_{n j+n} \mid \sigma\left(A_{n j}\right)\right)=H\left(A_{n j+n} \mid A_{n j}\right)
$$

for all $j$. On the other hand, $A$ (resp. $\sigma(A)$ ) is generated by the sequence $\left(A_{n j+n}\right)_{j}$ (resp. $\left.\left(\sigma\left(A_{n j}\right)\right)_{j}\right)$ with the commuting square condition

$$
E_{A_{n j}} E_{\sigma\left(A_{n j}\right)}=E_{\sigma\left(A_{n j-n}\right)} \text { for all } j \text {. }
$$


Hence by [10],

$$
H(A \mid \sigma(A))=\lim _{j \rightarrow \infty} H\left(A_{n j+n} \mid \sigma\left(A_{n j}\right)\right)=\lim _{j \rightarrow \infty} H\left(A_{n j+n} \mid A_{n j}\right) .
$$

Proof of Theorem 5. (1) By Lemma 6, Proposition 4 and Theorem 1,

$$
\begin{aligned}
H(A \mid \sigma(A)) & =\lim _{j \rightarrow \infty} H\left(A_{n j+n} \mid A_{n j}\right) \\
& =\lim _{k \rightarrow \infty} \frac{1}{k} \sum_{j=1}^{k+1} H\left(A_{n j+n} \mid A_{n j}\right) \\
& \leq \liminf _{k \rightarrow \infty} \frac{1}{k} \sum_{j=1}^{k+1}\left\{I\left(A_{n j+n}\right)-I\left(A_{n j}\right)\right\} \\
& =\liminf _{k \rightarrow \infty} \frac{1}{k} I\left(A_{n k+n}\right) \\
& \leq \lim _{k} \frac{1}{k} 2 H\left(A_{n k+n}\right) \\
& =2 H(\sigma) .
\end{aligned}
$$

(2) In [2], we proved that, if $\left(A_{j}\right)_{j}$ satisfies the bounded growth conditions, then for the number $m$ in the condition (ii)

$$
I\left(A_{j}\right)-I\left(A_{m}\right)=\sum_{i=m+1}^{j} H\left(A_{i} \mid A_{i-1}\right),
$$

and

$$
\lim _{j \rightarrow \infty} \frac{1}{j} \sum_{k \in K_{j}} t_{j}(k) d_{j}(k) \log t_{j}(k) d_{j}(k)=0,
$$

where $t_{j}$ is the trace vector of the restriction of $\tau$ to $A_{j}$.

This implies that

$$
\begin{aligned}
\lim _{j \rightarrow \infty} \frac{I\left(A_{j}\right)}{j} & =\lim _{j \rightarrow \infty} \frac{1}{j} \sum_{k \in K_{j}} t_{j}(k) d_{j}(k)\left[\log d_{j}(k)-\log t_{j}(k)\right] \\
& =\lim _{j \rightarrow \infty} \frac{2 H\left(A_{j}\right)}{j} .
\end{aligned}
$$

Hence,

$$
\begin{aligned}
H(A \mid \sigma(A)) & =\lim _{j} \frac{1}{j} \sum_{i} H\left(A_{n i+n} \mid A_{n i}\right)=\lim _{j} \frac{1}{j} I\left(A_{n j+n}\right) \\
& =\lim _{j} \frac{2}{j} H\left(A_{n j+n}\right)=2 H(\sigma) .
\end{aligned}
$$

By considering the standard tower

$$
N \subset M \subset M_{1} \subset M_{2} \subset \cdots \subset M_{n}=\left\langle M_{n-1}, e_{n-1}\right\rangle \subset \cdots
$$

obtained from the pair $N \subset M$ of $\mathrm{II}_{1}$-factors with $[M: N]<\infty$ by iterating the basic construction, it is proved in [11] that $H\left(M_{n} \mid N\right)=\log \left[M_{n}: N\right]$ if $H(M \mid N)=\log [M: N]$. Since the index has the multiplicative property [6], this implies that $H\left(M_{n} \mid N\right)=n H(M \mid N)$ if $H(M \mid N)=\log [M: N]$. The next corollary shows that a similar result holds for the pair $\sigma(M) \subset M$. 
Corollary 7. Let $a^{*}$-endomorphism $\sigma$ satisfy the same condition as in Theorem 5. Then for all $n$,

$$
H\left(A \mid \sigma^{n}(A)\right)=n H(A \mid \sigma(A)) .
$$

Proof. This is an immediate consequence of Theorem 5 and the fact $H\left(\sigma^{n}\right)=$ $n H(\sigma)$ by [2].

\section{6. $H(\sigma)$ AND $\lambda(A, \sigma(A))$ FOR $n$-SHIFT $\sigma$}

In this section, we shall investigate relations between the entropy $H(\sigma)$ and the constant $\lambda(A, \sigma(A))$ for an $n$-shift $\sigma$ of the tower $\left(A_{j}\right)_{j \in N}$ for a finite von Neumann algebra $A$ with a fixed faithful normal trace $\tau, \tau(1)=1$.

Definition 3. We shall call an increasing sequence $\left(A_{j}\right)_{j}$ of finite dimensional von Neumann subalgebras of a finite von Neumann algebra $A$ with a faithful normal trace $\tau$ a locally standard tower for $\alpha$ if thee exists a natural number $k$ which satisfies the following conditions:

(1) For a certain central projection $p_{k(j+1)}$ of $A_{k(j+1)}$, the inclusion matrix $\left[A_{j k} \hookrightarrow A_{k(j+1)} p_{k(j+1)}\right]$ is the transpose of $\left[A_{k(j-1)} \hookrightarrow A_{k j}\right]$, for each $j$.

(2) If $\left(t_{k(j-1)}(i)\right)_{i}$ is the trace vector for the restriction of $\tau$ to $A_{k(j-1)}$, then the value of $\tau$ of the minimal projections for $A_{k(j+1)} p_{k(j+1)}$ are given by $\left(\alpha t_{k(j-1)}(i)\right)_{i}$ for each $j$.

(3) There is $c>0$ such that $H\left(A_{2 k j}\right) \leq c-j \log \alpha$ for each $j$.

We call the number $2 k$ a period of the locally standard tower.

As examples of locally standard towers, we have the following:

(i) The tower $\left(A_{j}\right)_{j}$ in Example 1 is obviously a locally standard tower for $1 / m$, because the inclusion matrices in each step are all same.

(ii) The standard tower is a locally standard tower for $\left\|T^{t} T\right\|^{-1}$, because the inclusion matrix in the $j$ th step is the transpose of one in the $(j-1)$ th step for all $j$ [6]. Hence the tower $\left(A_{j}\right)_{j}$ is also locally standard if $A_{j+1}$ is a locally algebraic basic extension of $A_{j-1} \subset A_{j}$.

(iii) The tower $\left(A_{j}\right)_{j}$ in Example 2 is a locally standard tower for $\lambda$, because the central support of $e_{j}$ in $A_{j}$ satisfies the conditions (1) and (2) in Definition 3 and the condition (3) is proved by results in $\S 4.2$ and $\S 5.1$ in [6].

We shall treat another locally standard tower in the next section.

Theorem 8. Let $A$ be a finite von Neumann algebra with a fixed faithful normal trace $\tau, \tau(1)=1$. Let $\sigma$ be an $n$-shift on the locally standard tower $\left(A_{j}\right)_{j}$ for $\alpha$ with a period $2 n$, then

$$
H(A \mid \sigma(A)) \leq 2 H(\sigma) \leq-\log \alpha \leq \log \lambda(A, \sigma(A))^{-1} .
$$

Proof. Let $d_{j}$ and $t_{j}$ be the dimension vector of $A_{j}$ and the trace vector of the restriction of $\tau$ to $A_{j}$, respectively. Let $K_{j}$ be the set of simple summands of $A_{j}$. By the commuting square condition (3) in Definition 1 and [10],

$$
\lambda(A, \sigma(A))=\lim _{j \rightarrow \infty} \lambda\left(A_{n j+n}, \sigma\left(A_{n j}\right)\right) .
$$

Since the conditional expectations $E_{A_{n j}}$ and $E_{\sigma\left(A_{n j}\right)}$ are conjugate by an automorphism or antiautomorphism $\beta$ of $A_{n j+n}$, which satisfies the condition (4),

$$
\lambda\left(A_{n j+n}, \sigma\left(A_{n j}\right)\right)=\lambda\left(A_{n j+n}, A_{n j}\right) .
$$


On the other hand, since $\left(A_{j}\right)_{j}$ is a locally standard tower with a period $2 n$, by the same proof as Proposition 3 we have

$$
\lambda\left(A_{n j+n}, A_{n j}\right)^{-1} \geq \max _{k \in K_{n j-n}} \frac{t_{n j-n}(k)}{t_{n j+n}(k)}=\frac{1}{\alpha} .
$$

Hence,

$$
\log \lambda(A, \sigma(A))^{-1}=\lim _{j \rightarrow \infty} \log \lambda\left(A_{n j+n}, A_{n j}\right)^{-1} \geq-\log \alpha .
$$

On the other hand, by the condition (3) of the locally standard tower $\left(A_{j}\right)_{j}$ for $\alpha$, we have that

$$
H\left(A_{2 n j}\right) \leq c+j \log \frac{1}{\alpha} .
$$

Hence we have by Theorem 1,

$$
\log \lambda(A, \sigma(A))^{-1} \geq-\log \alpha \geq 2 \lim _{j \rightarrow \infty} \frac{1}{2 j} H\left(A_{2 n j}\right)=2 H(\sigma) .
$$

Combining with Theorem 5, we have

$$
H(A \mid \sigma(A)) \leq 2 H(\sigma) \leq-\log \alpha \leq \log \lambda(A, \sigma(A))^{-1} .
$$

The above proof shows that under a good condition, $\alpha=\lambda(A, \sigma(A))$. For example, if $\left(A_{j}\right)_{j}$ is periodic in the sense of [17], the equality holds. We shall show another example in $\S 7$.

The author would like to thank F. Hiai for pointing out a mistake in the proof of Theorem 8 in the preliminary version.

Corollary 9. Let $A$ be an injective finite factor with the canonical trace $\tau$ and $\sigma$ an $n$-shift of a locally standard tower for $A$ with a period $2 n$, then

$$
H(A \mid \sigma(A)) \leq 2 H(\sigma) \leq \log [A: \sigma(A)] .
$$

Proof. If $A$ is a factor, then $\sigma(A)$ is a subfactor of $A$, so that, by [1]?, $[A: \sigma(A)]=\lambda(A, \sigma(A))^{-1}$. Hence we have the corollary.

In the case that $\sigma(A)$ is a factor, it was determined in [10] when $H(A \mid \sigma(A))=$ $\log [A: \sigma(A)]$. In such a case, we have

$$
H(A \mid \sigma(A))=2 H(\sigma)=\log [A: \sigma(A)] .
$$

For example, the shifts $S$ in Example 1 and $\sigma$ for $\lambda>\frac{1}{4}$ in Example 2 satisfy the equality [2]. However, the shifts $\sigma$ in Example 2 have the following relation, [2]:

if $\lambda \leq \frac{1}{4}$.

$$
H(R \mid \sigma(R))=2 H(\sigma)<\log [R: \sigma(R)],
$$

\section{CANONICAL SHIFT}

In [9], Ocneanu defined a very nice *-endomorphism for the tower of the relative commutant algebras for the inclusion $N \subset M$ of type $\mathrm{II}_{1}$-factors with the finite index.

First we shall recall the definition and main properties of the canonical shift on the tower of relative commutants [9].

Let $M$ be a finite factor with the canonical trace $\tau$ and $N$ a subfactor of $M$ such that $[M: N]<+\infty$. Then the basic extension $M_{1}=\langle M, e\rangle$ is a $\mathrm{II}_{1}$ factor with the $\lambda=[M: N]^{-1}$-Markov trace [6] and there is a family $\left\{m_{i}\right\} \subset M$ 
which forms an "orthonormal basis" in $M$ with respect to the $N$ valued inner product $E_{N}\left(x y^{*}\right) \quad(x, y \in M)$, that is, each $x \in M$ is decomposed in the unique form as follows $[9,10]$ :

$$
x=\sum_{i} E_{N}\left(x m_{i}^{*}\right) m_{i} .
$$

Iterating the basic construction from $N \subset M$, we have the standard tower

$$
M_{-1}=N \subset M_{0}=M \subset M_{1}=\left\langle M_{0}, e_{0}\right\rangle \subset M_{2} \subset \cdots .
$$

Here, $e_{j}$ is the projection of $L^{2}\left(M_{j}, \tau_{j}\right)$ onto $L^{2}\left(M_{j-1}, \tau_{j-1}\right)$ and $\tau_{j}$ is the $\lambda$-Markov trace for $M_{j}$. Then from the family $\left(e_{j}\right)_{j}$ the projection $e(n, k)$ is obtained and

$$
M_{n-k} \subset M_{n} \subset M_{n+k}=\left\langle M_{n}, e(n, k)\right\rangle
$$

is an algebraic basic extension [9, 11]. Furthermore it is obtained in [9] that the "orthonormal basis" in $M_{n}$ with respect to $M_{n-k}$-valued inner product from the family of the basis in $\left(M_{j}\right)_{j}$.

Let $A_{j}=M^{\prime} \cap M_{j}$ for all $j$. The antiautomorphism $\gamma_{j}$ of $A_{2 j}=M^{\prime} \cap M_{2 j}$ defined by

$$
\gamma_{j}(x)=J_{j} x^{*} J_{j}, \quad x \in A_{2 j},
$$

is called the mirroring, where $J_{j}$ is the conjugate unitary on $L^{2}\left(M_{j}, \tau_{j}\right)$. Then for all $x \in M^{\prime} \cap M_{2 j}$, the following expression of the mirrorings is given:

$$
\gamma_{j}(x)=\left[M_{j}: M\right] \sum_{i} E\left(e m_{i}^{*} x\right) e m_{i},
$$

where $E$ is the conditional expectation of $M_{2 j}$ onto $M, e$ is the projection of $L^{2}\left(M_{j}\right)$ onto $L^{2}(M)$ and $\left(m_{i}\right)_{i}$ a module basis of $M_{j}$ over $M$. The expression implies that the mirrorings satisfy the following relation: $\gamma_{j+1} \cdot \gamma_{j}=\gamma_{j} \cdot \gamma_{j-1}$; for all $j \geq 1$ on $A_{2 j-2}$. In the view of this relation, the endomorphism $\Gamma$ of $\bigcup_{n} A_{n}$ can be defined by $\Gamma(x)=\gamma_{j+1}\left(\gamma_{j}(x)\right)$, for $x \in A_{2 j}$. Ocneanu called the endomorphism $\Gamma$ the canonical shift on the tower of the relative commutants. In the case of inclusions of infinite factors, similar ${ }^{*}$-endomorphisms are investigated by Longo [8]. The mapping $\Gamma$ has the following properties; for any $k, n \geq 0$ with $n \geq k, \Gamma\left(M_{k}^{\prime} \cap M_{n}\right)=M_{k+1}^{\prime} \cap M_{n+2}$.

Now, we shall consider the finite von Neumann algebra $A$ generated by the tower $\left(A_{j}\right)_{j}$ and extend $\Gamma$ to a trace preserving *-endomorphism of $A$ as follows.

Since $N \subset M$ are $\mathrm{II}_{1}$-factors with $[M: N]<+\infty$, there is a faithful normal trace on $\bigcup_{j} M_{j}$ which extends the canonical trace $\tau$ on $M$. We denote the trace by the same notation $\tau$.

Although $M_{j+1}$ is defined as a von Neumann algebra on $L^{2}\left(M_{j}, \tau_{j}\right)$, each $M_{j}$ can be considered as von Neumann algebras on the Hilbert space $L^{2}(M, \tau)$. Hence $\bigcup A_{j}$ and $\bigcup M_{j}$ can be considered as von Neumann algebras acting on $L^{2}(M, \tau)$. Let

$$
M_{\infty}=\left\{\bigcup_{j} M_{j}\right\}^{\prime \prime}, \quad A=\left\{\bigcup_{j} A_{j}\right\}^{\prime \prime} .
$$

Then $M_{\infty}$ is a finite factor with the canonical trace which is the extension of $\tau$. We denote it by the same notation $\tau$. Then $A$ is a von Neumann subalgebra 
of $M_{\infty}$. Since $\Gamma$ is a ultra-weakly continuous endomorphism of $\bigcup_{j} A_{j}, \Gamma$ is extended to a *-endomorphism of $A$.

Although, in the case discussed by Ocneanu, for all $k$, the mirroring $\gamma_{k}$ is a trace preserving map thanks to the assumption $N^{\prime} \cap M=\mathrm{C} 1$, in general, the mirrorings are not always trace preserving. However, the canonical shift is always trace preserving.

Lemma 10. For every $k, \gamma_{k+1} \cdot \gamma_{k}$ is a $\tau$-preserving isomorphism of $M^{\prime} \cap M_{2 k}$ onto $M_{2}^{\prime} \cap M_{2 k+2}$. Furthermore, if $E_{A_{1}}\left(e_{1}\right)=\lambda$ (for example $\left.N^{\prime} \cap M=\mathrm{C} 1\right)$, then $\gamma_{j}$ is a trace preserving antiautomorphism of $A_{2 j}$ for all $j$.

Proof. By the definition, it is obvious that

$$
\gamma_{k+1} \cdot \gamma_{k}\left(M^{\prime} \cap M_{2 k}\right)=\gamma_{k+1}\left(M^{\prime} \cap M_{2 k}\right)=M_{2}^{\prime} \cap M_{2 k+2} .
$$

In order to prove that $\tau\left(\gamma_{k+1} \cdot \gamma_{k}(x)\right)=\tau(x)$ for all $x \in M^{\prime} \cap M_{2 k}$, it is sufficient to prove that $\tau\left(\gamma_{k+1}(x)\right)=\tau\left(\gamma_{k}(x)\right)$, for all $x \in M^{\prime} \cap M_{2 k}$. Because of $[M: N]<\infty, M^{\prime} \cap B\left(L^{2}\left(M_{k}, \tau\right)\right)$ is a finite factor [6]. Let $\left(m_{i}\right)_{i}$ be an "orthonormal basis" in $M_{k+1}$ with respect to the $M_{k}$-valued inner product $E_{M_{k}}\left(x y^{*}\right)$, for $x, y \in M_{k+1}$. Every $\xi \in L^{2}\left(M_{k+1}, \tau\right)$ is written in the form $\xi=\sum_{i} \xi_{i} m_{i}\left(\xi_{i} \in L^{2}\left(M_{k}, \tau\right)\right)$. We shall embed an $x \in B\left(L^{2}\left(M_{k}, \tau\right)\right)$ into $B\left(L^{2}\left(M_{k+1}, \tau\right)\right.$ by $x \xi=\sum_{i} x\left(\xi_{i}\right) m_{i}$. Then $M^{\prime} \cap B\left(L^{2}\left(M_{k}, \tau\right)\right)$ is considered as a subfactor (with the canonical trace $\psi$ ) of the finite factor $M^{\prime} \cap B\left(L^{2}\left(M_{k+1}, \tau\right)\right)$ with the canonical trace $\phi$. Hence, for an $x \in M^{\prime} \cap M_{2 k} \subset M^{\prime} \cap B\left(L^{2}\left(M_{k}, \tau\right)\right)$, we have

$$
\tau\left(\gamma_{k}(x)\right)=\psi(x)=\phi(x)=\tau\left(\gamma_{k+1}(x)\right) .
$$

Assume that $E_{A_{1}}\left(e_{1}\right)=\lambda=[M: N]^{-1}$. Then by [9 and 10], this implies that

$$
\tau_{2 j+2}(x)=\tau_{M^{\prime} \cap B\left(L^{2}\left(M_{j+1}, \tau_{j+1}\right)\right)}(x)
$$

for all $x \in M^{\prime} \cap M_{2 j+2}$, where $\tau_{i}$ (resp. $\tau_{L}$ ) is the canonical trace of $M_{i}$ (resp. factor $L$ ). Let $x \in M^{\prime} \cap M_{2 j}$. Since $M^{\prime} \cap M_{2 j} \subset M^{\prime} \cap M_{2 j+2}$,

$$
\tau_{2 j+2}\left(\gamma_{j+1}(x)\right)=\tau_{M^{\prime} \cap B\left(L^{2}\left(M_{j+1}, \tau_{j+1}\right)\right.}(x) .
$$

This implies that

$$
\tau_{2 j+2}\left(\gamma_{j+1}(x)\right)=\tau_{2 j+2}(x) .
$$

Thus the mirroring $\gamma_{j+1}$ is a trace preserving antiautomorphism of $A_{2 j+2}$.

By Lemma 10, the canonical shift $\Gamma$ on the tower of the relative commutants $\left(A_{j}\right)_{j}$ of $M$ is extended to a $\tau$-preserving *-endomorphism of $A$. We call the *-endomorphism of $A$ the canonical shift for the inclusion $M \supset N$ and denote it by the same notation $\Gamma$.

We will show the canonical shift $\Gamma$ is a 2-shift on the tower $\left(A_{j}\right)_{j}$ for $A$.

Lemma 11. Let $L$ be a finite von Neumann algebra with a faithful normal trace $\tau, \tau(1)=1$. If $M$ is a subfactor of $L$, then

$$
\tau(x y)=\tau(x) \tau(y) \quad\left(x \in M, y \in M^{\prime} \cap L\right) .
$$

Proof. Let $E$ be the conditional expectation of $L$ onto $M$ conditioned by $\tau$. For $x \in M$ and $y \in M^{\prime} \cap L$,

$$
E_{M}(y) x=E_{M}(y x)=E_{M}(x y)=x E_{M}(y),
$$


which implies $E_{M}(y) \in M^{\prime} \cap M$. Since $M$ is a factor, $E_{M}(y)=\tau(y)$. Hence

$$
\tau(x y)=\tau\left(E_{M}(x y)\right)=\tau\left(x E_{M}(y)\right)=\tau(x) \tau(y) .
$$

Proposition 12. The canonical shift $\Gamma$ for the inclusion $N \subset M$ satisfies the conditions (1), (2) and (3) for 2-shifts. If $E_{A_{1}}\left(e_{1}\right)=[M: N]^{-1}$, then $\Gamma$ is a 2-shift on the tower $\left(A_{j}\right)_{j}$ for $A$.

Proof. Since $[M: N]<+\infty$, for all $j, A_{j}=M^{\prime} \cap M_{j}$ is finite dimensional [6]. For all natural numbers $j$ and $k$,

$$
\Gamma^{k}\left(A_{j}\right)=\Gamma^{k}\left(M^{\prime} \cap M_{j}\right)=M_{2 k}^{\prime} \cap M_{j+2 k} .
$$

This implies

$$
\left\{A_{j}, \Gamma\left(A_{j}\right), \ldots, \Gamma^{m}\left(A_{j}\right)\right\}^{\prime \prime} \subset M^{\prime} \cap M_{j+2 m}=A_{j+2 m} .
$$

For each $j$, let $k_{j}=\left[\frac{j}{2}\right]+1$. If $m \geq k_{j}$, then

$$
\Gamma^{m}\left(A_{j}\right)=M_{2 m}^{\prime} \cap M_{j+2 m} \subset A_{j}^{\prime} .
$$

Combining this with Lemma 11 , we have that $\left(k_{j}\right)_{j}$ satisfies the condition (2) for 2-shifts. It is proved in [13] that $E_{M_{k}^{\prime} \cap M_{j}} E_{M_{i}}=E_{M_{k}^{\prime} \cap M_{k}}$, for $k \leq i \leq j$. This implies that

$$
\begin{aligned}
E_{A_{j}} E_{\Gamma\left(A_{j}\right)} & =E_{M^{\prime} \cap M_{j}} E_{M_{2}^{\prime} \cap M_{j+2}}=E_{M^{\prime} \cap M_{j}} E_{M_{j}} E_{M_{2}^{\prime} \cap M_{j+2}} \\
& =E_{M^{\prime} \cap M_{j}} E_{M_{2}^{\prime} \cap M_{j}}=E_{\Gamma\left(A_{j-2}\right)} .
\end{aligned}
$$

Hence $\Gamma$ satisfies (1), (2), and (3) in Definition 1 for $n=2$.

Assume that $E_{A_{1}}\left(e_{1}\right)=[M: N]^{-1}$. Then by Lemma 10, the mirroring $\gamma_{j+1}$ is a trace preserving antiautomorphism of $A_{2 j+2}$. Since $\Gamma\left(A_{2 j}\right)=\gamma_{j+1}\left(A_{2 j}\right), \Gamma$ is a 2 -shift on the tower $\left(A_{j}\right)_{j}$.

Next, we shall show the entropy $H(\Gamma)$ of the *-endomorphism $\gamma$ of $A$ is always dominated by $\log [M: N]$.

Lemma 13. Let $B=A \cap N$ for von Neumann subalgebras $A$ and $N$ of a finite von Neumann algebra $M$ satisfying the commuting square condition: $E_{A} E_{N}=$ $E_{N} E_{A}=E_{B}$. Then, $H(M \mid N) \geq H(A \mid B), \lambda(M, N) \leq \lambda(A, B)$.

Proof. By the commuting square condition, we have $E_{N}(x)=E_{B}(x)$ for all $x \in A$. Hence

$$
\begin{aligned}
H(M \mid N) & =\sup _{x \in S_{1} \cap M} \sum_{i}\left[\tau \eta E_{N}\left(x_{i}\right)-\tau \eta\left(x_{i}\right)\right] \\
& \geq \sup _{x \in S_{1} \cap A} \sum_{i}\left[\tau \eta E_{N}\left(x_{i}\right)-\tau \eta\left(x_{i}\right)\right]=H(A \mid B),
\end{aligned}
$$

and

$$
\begin{aligned}
\lambda(M, N) & =\max \left\{\lambda: E_{N}(x) \geq \lambda x, x \in M_{+}\right\} \\
& \leq \max \left\{\lambda: E_{B}(x) \geq \lambda x, x \in A_{+}\right\}=\lambda(A, B) .
\end{aligned}
$$

Let $B$ and $C$ be the von Neumann subalgebras of $A$ defined by

$$
B=\left(\bigcup_{j}\left(M_{1}^{\prime} \cap M_{j}\right)\right)^{\prime \prime}, \quad C=\left(\bigcup_{j}\left(M_{2}^{\prime} \cap M_{j}\right)\right)^{\prime \prime} \text {. }
$$


Theorem 14. Let $\Gamma$ be the canonical shift for the inclusion $N \subset M$ of type $\mathrm{II}_{1}$-factors with $[M: N]<\infty$. Then

If $E_{A_{1}}\left(e_{1}\right)=[M: N]^{-1}$, then

$$
H(\Gamma)=\lim _{k \rightarrow \infty} \frac{H\left(M^{\prime} \cap M_{2 k}\right)}{k} .
$$

$$
H(A \mid C) \leq 2 H(\Gamma) \leq \log \lambda(A, C)^{-1}=2 H(M \mid N)=2 \log [M: N] .
$$

Proof. The shift $\Gamma$ satisfies conditions (1) and (2) for 2-shifts. Hence by Theorem 1 ,

$$
H(\Gamma)=\lim _{k \rightarrow \infty} \frac{H\left(A_{2 k}\right)}{k} .
$$

Assume that $E_{A_{1}}\left(e_{1}\right)=[M: N]^{-1}$. Then the canonical shift $\Gamma$ is a 2-shift on the tower $\left(A_{j}\right)_{j}$ of the relative commutants of $M$ by Proposition 12. For the projection $e_{j}$ of $L^{2}\left(M_{j}, \tau\right)$ onto $L^{2}\left(M_{j-1}, \tau\right)$, let $p_{j}$ be the central support of $e_{j}$ in $A_{j}$. Then, for all $j \geq 1$,

$$
A_{j-1} \subset A_{j} \subset A_{j+1} p_{j+1}
$$

is an algebraic basic extension for $A_{j-1} \subset A_{j}$ and the trace vectors of $A_{j-1}$ and $A_{j+1}$ satisfy the condition (2) in Definition 3 for $\lambda=[M: N]^{-1},[5,9$, $13,17]$. On the other hand, [10, Theorem 4.4] assures that for all $j$,

Since

$$
2 H\left(M^{\prime} \cap M_{j}\right) \leq H\left(M_{j} \mid M\right) \text {. }
$$

$$
H\left(M_{j} \mid M\right) \leq \log \left[M_{j}: M\right]=-j \log \lambda,
$$

by [10 and 11], the condition (3) in Definition 3 for $\left(A_{j}\right)$ is satisfied. Hence the sequence $\left(A_{j}\right)_{j}$ is a locally standard tower for $\lambda^{2}$ with period 4 . Hence, by Theorem 8,

$$
H(A \mid \Gamma(A)) \leq 2 H(\Gamma) \leq 2 \log [M: N] \leq \log \lambda(A, \Gamma(A))^{-1} .
$$

Since $\Gamma\left(M_{k}^{\prime} \cap M_{j}\right)=M_{k+2}^{\prime} \cap M_{j+2}$, we have $\Gamma(A)=C$. Hence,

$$
H(A \mid C) \leq 2 H(\Gamma) \leq 2 \log [M: N] \leq \log \lambda(A, C)^{-1} .
$$

Every factor $M_{j}$ can be considered as a von Neumann algebra on $L^{2}(M, \tau)$ by Jones' method [6]. Then as von Neumann algebras on $L^{2}(M, \tau)$, for all $j$,

$$
E_{M^{\prime} \cap M_{j}} E_{M_{2}^{\prime}}=E_{M_{2}^{\prime}} E_{M^{\prime} \cap M_{j}}=E_{M_{2}^{\prime} \cap M_{j}}
$$

Since $A$ is generated by the tower $\left(M^{\prime} \cap M_{j}\right)_{j}$ and $C$ is generated by the tower $\left(M_{2}^{\prime} \cap M_{j}\right)_{j}$, it follows that $E_{A} E_{M_{2}^{\prime}}=E_{C}$, where all algebras are considered as von Neumann subalgebras of a finite factor $M^{\prime}$ on $L^{2}(M, \tau)$. By Lemma 13, this implies $\lambda\left(M_{2}^{\prime}, M^{\prime}\right) \leq \lambda(A, C)$. Since $M$ and $N$ are factors, $\lambda(M, N)^{-1}=$ $[M: N]$. On the other hand, Jones proved that $M^{\prime} \supset M_{2}^{\prime}$ are finite factors with $\left[M^{\prime}: M_{2}^{\prime}\right]=\left[M_{2}: M\right]=[M: N]^{2}$. Hence

$$
\lambda(A, \Gamma(A))^{-1}=\lambda(A, C)^{-1}=2[M: N] .
$$

The condition that $E_{A_{1}}\left(e_{1}\right)=[M: N]^{-1}$ is equivalent to $H(M \mid N)=\log [M: N]$ [10]. Hence we have

$$
H(A \mid C) \leq 2 H(\Gamma) \leq \log \lambda(A, C)^{-1}=2 \log [M: N]=2 H(M \mid N) .
$$

The above simple proof, where the condition (3) in Definition 3 for the sequence $\left(A_{j}\right)_{j}$ was used, was indicated by F. Hiai.

As an immediate consequence, we have 
Corollary 15. Under the same conditions as in Theorem 14, let $A$ be a factor. Then

$$
H(A \mid C) \leq 2 H(\Gamma) \leq 2 \log [A: B]=2 \log [M: N] .
$$

Corollary 16. Let $\Gamma$ be the canonical shift for the inclusion $N \subset M$ of type $\mathrm{II}_{1}$-factors with $[M: N]<\infty$. If $N^{\prime} \cap M=\mathbf{C} 1$, then

$$
H(\Gamma) \leq H(M \mid N)=\log [M: N] .
$$

For a pair $N \subset M$ of hyperfinite type $\mathrm{II}_{1}$-factors with $[M: N]<\infty$, Popa says that $N \subset M$ has the generating property if there exists a choice of the standard tunnel of subfactors $\left(N_{j}\right)_{j}$ such that $M$ is generated by the increasing sequence $\left(N_{j}^{\prime} \cap M\right)_{j}$.

Corollary 17. Assume that $N \subset M$ has the generating property. If $E_{N^{\prime} \cap M}\left(e_{0}\right)=$ $[M: N]^{-1}$, then

$$
H(M \mid N)=H(\Gamma)=\log [M: N] .
$$

Proof. By [6], we consider all $M_{j}$ as factors acting on $L^{2}(M, \tau)$. Let $J$ be the canonical conjugation on $L^{2}(M, \tau)$. For each $j$, let $N_{j}=J M_{j}^{\prime} J$. Then the mapping $\Phi$ defined by $\Phi(x)=J x J$ is a trace preserving anti-isomorphism [13] such that $\Phi(A)=\left(\bigcup_{j}\left(N_{j}^{\prime} \cap M\right)\right)^{\prime \prime}$ and $\Phi(B)=\left(\bigcup_{j}\left(N_{j}^{\prime} \cap N\right)\right)^{\prime \prime}$ because $E_{N^{\prime} \cap M}\left(e_{0}\right)=[M: N]^{-1}$. Although, the tunnel of subfactors is not uniquely determined, the pair of algebras of relative commutants is unique up to isomorphism [13], that is, let $M \supset N \supset N_{1} \supset \cdots$ and $M \supset N \supset P_{1} \supset \cdots$ be two choices of the standard tunnels, then there exists a trace preserving isomorphism $\Psi$ such that

$$
\Psi\left(\left(\bigcup_{j}\left(N_{j}^{\prime} \cap M\right)\right)^{\prime \prime}\right)=\left(\bigcup_{j}\left(P_{j}^{\prime} \cap M\right)\right)^{\prime \prime}
$$

and

$$
\Psi\left(\left(\bigcup_{j}\left(N_{j}^{\prime} \cap N\right)\right)^{\prime \prime}\right)=\left(\bigcup_{j}\left(P_{j}^{\prime} \cap N\right)\right)^{\prime \prime} .
$$

Since $N \subset M$ has the generating property, we have a trace preserving antiautomorphism of $M$ onto $A$ which transpose $N_{1}$ onto $C$. Hence $H(A \mid C)=$ $H\left(M \mid N_{1}\right)$. If $E_{N^{\prime} \cap M}\left(e_{0}\right)=[M: N]^{-1}$, then $H\left(M \mid N_{1}\right)=\log \left[M: N_{1}\right]$ [11]. Hence by Theorem 14 ,

$$
H(M \mid N)=H(\Gamma)=\log [M: N] .
$$

As a sufficient condition for the two assumptions in Corollary 17, Ocneanu [9] introduced the following notion for a pair $N \subset M$ with $N^{\prime} \cap M=\mathrm{Cl}$, and Popa [13] extended it to general cases. The inclusion $N \subset M$ of type $\mathrm{II}_{1}$ factors with $[M: N]<+\infty$ is said to have the finite depth if $\sup _{j}\left(k_{j}\right)<+\infty$, where $k_{j}$ is the cardinal number of simple summands of $M^{\prime} \cap M_{j}$.

Remark 18. If the inclusion $N \subset M$ of type $\mathrm{II}_{1}$-factors with the finite index and finite depth, then the tower $\left(A_{j}\right)_{j}$ of relative commutants satisfies the bounded growth conditions.

If an inclusion $N \subset M$ has the finite depth, then $E_{N^{\prime} \cap M}\left(e_{0}\right)=[M: N]^{-1}$ and $N \subset M$ has the generating property [13]. Hence we have 
Corollary 19. Let $N \subset M$ be type $\mathrm{II}_{1}$-factors with the finite index and the finite depth. Let $\Gamma$ be the canonical shift for $N \subset M$. Then

$$
H(M \mid N)=H(\Gamma)=\log [M: N] .
$$

Remark 20. In Corollary 18, the shift $\Gamma$ is considered as an *-endomorphism of the algebra $A$ generated by the tower $\left(A_{j}\right)_{j}$ of the relative commutants of $M$. Since $N \subset M$ has the finite depth, the shift $\Gamma$ induces a trace preserving *-endomorphism of $M$ which sending $M$ to the subfactor $P$ in such a way that $P \subset N \subset M$ is the algebraic basic extension for $P \subset N$. Then the *-endomorphism of $M$ has the same property as $\Gamma$.

In the rest of this section, we shall show that the canonical shift has an ergodic property, which is similar to the canonical endomorphism in [7]. Therefore the canonical shift is a shift in the sense due to Powers [14].

Proposition 21. Let $N \subset M$ be type $\mathrm{II}_{1}$-factors with the finite index. Then the canonical shift $\Gamma$ for $N \subset M$ satisfies that

$$
\bigcap_{k} \Gamma^{k}(A)=\mathbf{C} 1 \text {. }
$$

Proof. The von Neumann algebra $A$ is contained in the type $\mathrm{II}_{1}$-factor $M_{\infty}=$ $\left.\left(\bigcup_{j} M_{j}\right)\right)^{\prime \prime}$ with the canonical trace $\tau$ which is the extension of $\tau$. Let take an $x \in \bigcap_{x} \Gamma^{k}(A)$. For any $\varepsilon>0$, there exists an integer $k$ such that $\left\|x-x_{k}\right\|_{2}<\varepsilon$ for some $x_{k} \in A_{k}$. Let $E$ be the conditional expectation of $M_{\infty}$ onto $M_{k}$. Since $x \in \Gamma^{k}(A) \subset M_{k}^{\prime} \cap M_{\infty}$, for any $y \in M_{k}, E(x) y=E(x y)=y E(x)$. This implies $E(x) \in M_{k} \cap M_{k}^{\prime}$, that is, $E(x)=\tau(x)$. On the other hand, $x_{k} \in M_{k}$. Hence

$$
\|x-\tau(x)\|_{2} \leq\left\|x-x_{k}\right\|_{2}+\left\|x_{k}-E(x)\right\|_{2}<2 \varepsilon .
$$

This means, $x \in \mathbf{C} 1$.

\section{EXTENSION OF CANONICAL SHIFT}

In this section, we shall show that the canonical shift $\Gamma$ is extended to an ergodic *-automorphism $\Theta$ of a larger von Neumann algebra in such a way that $H(\Gamma)=H(\Theta)$.

Let $N \subset M$ be type $\mathrm{II}_{1}$-factors with $[M: N]<\infty$. Let

$$
M_{-1}=N \subset M=M_{0} \subset M_{1}=\langle M, e\rangle \subset \cdots \subset M_{j}=\left\langle M_{j-1} e_{j-1}\right\rangle \subset \cdots
$$

be the standard tower obtained from $N \subset M$. Let $M_{\infty}$ be the finite factor generated by the tower $\left(M_{j}\right)_{j}$.

Proposition 22. Let $N \subset M$ be type $\mathrm{II}_{1}$-factors with the finite index and $\tau$ the canonical trace of $M$. Let $\sigma$ be $a^{*}$-isomorphism of $M$ onto $N$. Then the following statements are equivalent:

(1) There exists $a^{*}$-isomorphism $\sigma_{1}$ of $M_{1}$ onto $M$ such that for all $x \in M$, $\sigma_{1}(x)=\sigma(x)$.

(2) There exists a projection $e \in M$ such that $\sigma(N)=\{e\}^{\prime} \cap N$ and $E_{N}(e)=$ $\lambda 1=[M: N]^{-1}$.

(3) There exists a projection $e \in M$ such that for all $y \in N$, eye $=E_{\sigma(N)}(y) e$, $\tau(e y)=\lambda \tau(y)$, and $M$ is generated by $N$ and $e$ as a von Neumann algebra. 
(4) There exists an automorphism $\Theta$ on $M_{\infty}$ such that for all $x \in M$ and all $j, \boldsymbol{\Theta}(x)=\sigma(x)$ and $\Theta\left(e_{j}\right) \in M_{j}$.

(5) The decreasing sequence $M \supset N \supset \sigma(N) \supset \cdots \supset \sigma^{j}(N) \supset \cdots$ is a standard tunnel.

Proof. (1) $\Rightarrow(2)$. Let $e=\sigma_{1}\left(e_{0}\right)$, where $e_{0}$ is the projection of $L^{2}(M, \tau)$ onto $L^{2}(N, \tau)$. Since $\sigma$ must be $\tau$-preserving, for all $x \in M$,

$$
\sigma\left(E_{M}(x)\right)=E_{\sigma(M)}(\sigma(x)) .
$$

By [6], $E_{M}\left(e_{0}\right)=\lambda 1$ and $N=\left\{e_{0}\right\}^{\prime} \cap M$. Hence (2) holds.

(2) $\Rightarrow(3)$. The projection $e$ in (2) satisfies that $e y e=E_{\sigma(N)}(y) e$ for all $y \in N$ and $M=\{N, e\}^{\prime \prime}$ by [11]. If $y \in N$, then

$$
\tau(e y)=\tau\left(E_{\sigma(N)}(y) e\right)=\tau\left(E_{\sigma(N)}(y) E_{N}(e)\right)=\lambda \tau(y) .
$$

(3) $\Rightarrow(1)$. We put

$$
\sigma_{1}\left(\sum_{I=1}^{k} a_{i} e_{0} b_{i}\right)=\sum_{i=1}^{k} \sigma\left(a_{i}\right) e \sigma\left(b_{i}\right),
$$

for $a_{i}, b_{i} \in M$. The map $\sigma$ is a well-defined *-homomorphism.

In fact, assume $z=\sum_{i} a_{i} e_{0} b_{i}=0$. Since $\sigma$ is trace preserving,

$$
\begin{aligned}
\|z\|_{2}^{2} & =\sum_{i, j} \tau\left(b_{i}^{*} e_{0} a_{i}^{*} a_{j} e_{0} b_{j}\right) \\
& =\sum_{i, j} \tau\left(e_{0} E_{N}\left(a_{i}^{*} a_{j}\right) E_{N}\left(b_{j} b_{i}^{*}\right)\right) \\
& =\lambda \sum_{i, j} \tau\left(E_{\sigma(N)}\left(\sigma\left(a_{i}^{*}\right) \sigma\left(a_{j}\right)\right) E_{\sigma(N)}\left(\sigma\left(b_{j}\right) \sigma\left(b_{i}^{*}\right)\right)\right) \\
& =\sum_{i, j} \tau\left(e E_{\sigma(N)}\left(\sigma\left(a_{i}\right)^{*} \sigma\left(a_{j}\right)\right) \sigma\left(b_{j}\right) \sigma\left(b_{i}^{*}\right)\right) \\
& =\left\|\sum_{i} \sigma\left(a_{i}\right) e \sigma\left(b_{i}\right)\right\|_{2}^{2}
\end{aligned}
$$

Thus $\sigma_{1}$ is extended to a *-isomorphism of $M_{1}$ onto $M$. By the definition, for all $a \in M, \sigma(a)=\sigma_{1}(1)=\sigma_{1}(a)=\sigma_{1}(1) \sigma(a)$ and $e \sigma_{1}(1)=\sigma_{1}\left(e_{0}\right)=$ $\sigma_{1}(1) e$, because $\sigma_{1}$ is a ${ }^{*}$-isomorphism of $M_{1}$ onto $M$. Since the factor $M$ is generated by $N$ and $e$, the projection $\sigma_{1}(1)=1$. Hence for all $x \in M$, $\sigma_{1}(x)=\sigma_{1}(x 1)=\sigma(x)$.

(1) $\Rightarrow(4)$. Let us consider the ${ }^{*}$-isomorphism $\sigma_{1}$ of $M_{1}$ onto $M$ such that $\sigma_{1}(x)=\sigma(x)$ for all $x \in M$. Then the projection $e_{0} \in M_{1}$ satisfies that $\sigma_{1}(M)=N=e_{0}^{\prime} \cap M$ and $E_{M}\left(e_{0}\right)=\lambda 1$. Hence the above discussion implies that there exists a *-isomorphism $\sigma_{2}$ of $M_{2}$ onto $M_{1}$ such that $\sigma_{2}(x)=$ $\sigma_{1}(x)$ for $x \in M_{1}$. Iterating this method, we have the sequence $\left(\sigma_{j}\right)_{j}$ of ${ }^{*}$ isomorphisms of $M_{j}$ onto $M_{j-1}$ such that $\sigma_{j}(x)=\sigma_{j-1}(x)$ for $x \in M_{j-1}$. For any $y \in \bigcup_{j} M_{j}$, let $\Theta(y)=\sigma_{j}(y)$ if $y \in M_{j}$. Then $\theta$ is extended to the (we denote it by the same notation $\Theta$ ) mapping of $M_{\infty}$. The mapping $\Theta$ is an automorphism and $\boldsymbol{\Theta}^{\prime}(x)=\tau(x)$ for $x \in M_{\infty}$ and $\Theta\left(e_{j}\right)=\sigma\left(e_{j}\right) \in M_{j}$. 
(4) $\Rightarrow(1)$. The automorphism $\Theta$ satisfies that $\Theta(M)=N$ and $\Theta\left(e_{0}\right) \in M$. Hence $\Theta$ is an automorphism of $M_{1}$ onto $M$ such that $\Theta(x)=\sigma(x)$ for $x \in M$.

(3) $\Rightarrow(5)$. Let us take such a projection $e$ as in (3). If $z \in \sigma(N)$ satisfies $z e=0$, then $0=\|e z\|_{2}=\lambda\|z\|_{2}$. Hence $z=0$. Clearly, $M$ is an algebraic basic extension for $\sigma(N) \subset N$. Let $\sigma^{i}(e)=e_{-i}$ and $N_{i}=\sigma^{i}(N)$. Then $N_{i} \supset N_{i+1} \supset N_{i+2}$ is an algebraic basic extension for $N_{i+1} \supset N_{i+2}$.

$(5) \Rightarrow(3)$. Since the tunnel is standard, there is the basic projection $e \in M$ for $\sigma(N) \subset N$. The projection $e$ satisfies the conditions (3).

Definition 4. Let $\sigma$ be a ${ }^{*}$-isomorphism of a type $\mathrm{II}_{1}$-factor $M$ onto a subfactor $N$ with the finite index. If $\sigma$ satisfies the equivalent conditions in Proposition 22, then we call $\sigma$ a basic ${ }^{*}$-endomorphism for the inclusion $N \subset M$.

Let $\sigma$ be a basic *-endomorphism of the inclusion $N \subset M$ of type $\mathrm{II}_{1}$ factors with the finite index. Let $P_{j}=M \cap \sigma^{j}(M)^{\prime}$. Then $\left(P_{j}\right)_{j}$ is an increasing sequence of finite dimensional von Neumann algebras. Let $P$ be the von Neumann algebra generated by $\left(P_{j}\right)_{j}$. Then $P$ is a von Neumann subalgebra of $M$ and we have the following

Proposition 23. Let $\sigma$ be a basic *-endomorphism for the inclusion $N \subset M$ of type $\mathrm{II}_{1}$-factors with the finite index. Then,

$$
H(\sigma)=\lim _{k \rightarrow \infty} \frac{H\left(M \cap \sigma^{k}(M)^{\prime}\right)}{k} .
$$

Assume that $E_{N^{\prime} \cap M}(e)=[M: N]^{-1}$ for a basic projection of $\sigma(N) \subset N$. Then $\sigma^{m}$ is a m-shift on the tower $\left(P_{j}\right)_{j}$ for $P$ for all even number $m$ and satisfies the following relations. For all even $m$,

$$
H\left(P \mid \sigma^{m}(P)\right) \leq 2 m H(\sigma) \leq \log \lambda\left(P, \sigma^{m}(P)\right)^{-1}=m \log [M: N] .
$$

Proof. The condition (1) is obviously satisfied. For every $j$, put $k_{j}=\left[\frac{j}{n}\right]+1$. Then by Lemma 11, (2) for $n$-shift is satisfied. Hence we have the first equality. Since $\left(\sigma^{j}(M)\right)_{j}$ is a standard tunnel, $\left(\sigma^{j n}(M)\right)_{j}$ is a standard tunnel. Hence the commuting square condition (3) is satisfied [13]. We take the mirroring $\gamma$ defined by the conjugation on $L^{2}\left(\sigma^{n(j+1)}(M)\right)$. Then by a similar method as in the proof of Lemma 10, $\gamma$ is the trace preserving antiautomorphism of $P_{2 n(j+1)}$ such that $\gamma\left(P_{2 n j}\right)=\sigma^{2 n}\left(P_{2 n j}\right)$, because $\sigma$ is a basic *-endomorphism. Hence $\sigma^{2 n}$ is an $2 n$-shift on the tower $\left(P_{j}\right)_{j}$ for $P$, and by Theorem 8 and [2], for all $n$,

$$
H\left(P \mid \sigma^{2 n}(P)\right) \leq 2 H\left(\sigma^{2 n}\right)=4 n H(\sigma) .
$$

Let $p_{j}$ be the central support of the projection $e_{-j}$ in $P_{j}$ which satisfies that $\sigma^{j}(M)=e_{-j}^{\prime} \cap \sigma^{j-2}(M)$. Then the inclusion $P_{j+1} \subset P_{j+2} p_{j+2}$ is an algebraic basic construction corresponding to $P_{j} \subset P_{j+1}$ via $P_{j+1} \simeq P_{j+2} p_{j+2}$. This means that $\left(P_{j}\right)_{j}$ is a locally standard tower with a period 2 , for $\lambda=[M: N]^{-1}$ that is, with every even number as a period. Hence

$$
2 H\left(\sigma^{m}\right) \leq \log \lambda\left(P, \sigma^{m}(P)\right)^{-1},
$$

for all even $m$. Since $E_{P} E_{\sigma^{n}(M)}=E_{\sigma^{n}(P)}$, by Lemma 13,

$$
\left.\log \lambda\left(P, \sigma^{n}(P)\right)^{-1} \leq \log \lambda\left(M, \sigma^{n}(M)\right)^{-1}=\log \left[M: \sigma^{n}(M)\right)\right]=n \log [M: N] .
$$

Thus we have the stated inequality. 
Corollary 24. Let $\sigma$ be the same as in Proposition 23. Then

$$
2 H(\sigma) \leq \log [M: N] .
$$

Furthermore, if the inclusion $N \subset M$ has finite depth, then

$$
H(M \mid N)=2 H_{M}(\sigma)=2 H(\sigma)=\log [M: N],
$$

where $H_{M}(\sigma)$ is the entropy of $\sigma$ as $a^{*}$-endomorphism of $M$.

Proof. The first inequality is clear by Proposition 23. Assume that the inclusion $N \subset M$ has finite depth. Then it is proved in [13] that there exists a choice of the standard tunnel $\left(N_{i}\right)_{i}$ such that $M$ is generated by $\left\{N_{i}^{\prime} \cap M\right\}_{i}$. Since $\left(\sigma^{i}(M)\right)_{i}$ is also a standard tunnel of subfactors, there exists a trace preserving *-isomorphism of $M$ onto $P$ carrying $N$ onto $\sigma(P)$ [13]. The finite depth assumption implies that $E_{N^{\prime} \cap M}\left(e_{N}\right)=1 /[M: N]$ by [13]. Hence $\log [M: N]=$ $H(M \mid N)$ by [10]. On the other hand, $H\left(M \mid \sigma^{n}(M)\right)=H\left(P \mid \sigma^{n}(P)\right)$ for all $n$, because $\sigma$ is a trace preserving ${ }^{*}$-endomorphism of $M$. Hence

$$
H\left(M \mid \sigma^{n}(M)\right)=\log \left[M: \sigma^{n}(M)\right]=n \log [M: N] .
$$

By Proposition 23,

$$
H(M \mid N)=2 H_{M}(\sigma)=2 H(\sigma)=\log [M: N] .
$$

As an example of a basic *-endomorphism, we have the *-endomorphism $\sigma$ in Example 2.

We shall show that another typical example of a basic *-endomorphism is the canonical shift on the tower of relative commutants in $\S 7$.

Proposition 25. Let $M \supset N$ be type $\mathrm{II}_{1}$-factors with the finite index and finite depth. Then the canonical shift $\Gamma$ for the inclusion $M \supset N$ is a basic *endomorphism of $A=\left(\bigcup_{j}\left(M^{\prime} \cap M_{j}\right)_{j}\right)^{\prime \prime}$.

Proof. If $M \supset N$ has finite index and finite depth, then $A$ is a finite factor which is anti-isomorphic to $M$. Let $C$ be the subfactor $\Gamma(A)$. Then $[A$ : $C]=[M: N]^{2}$. To prove that $\Gamma$ is the basic ${ }^{*}$-endomorphism of $A$, we have to show the existence of a projection in $A$ which satisfies the statement (2) in Proposition 22. Let $f$ be a projection in $M_{4}$ such that $M_{4}$ is generated by $M_{2}$ and $f$. Then

$$
M_{4}^{\prime} \cap M_{j}=\{f\}^{\prime} \cap M_{2}^{\prime} \cap M_{j}
$$

for all $j \geq 4$. By the definition of $A$ and the property of $\Gamma, \Gamma(C)=\{f\}^{\prime} \cap C$. Since $f$ is the basic projection for the standard tower $M \subset M_{2} \subset M_{4}$ and $N \subset M$ has finite depth, by [13],

$$
E_{C}(f)=[M: N]^{2}=[A: C] .
$$

In [2], we proved that some kinds of *-endomorphisms are extended to ergodic *-automorphisms of larger algebras with same values as entropies. Here we shall show this also holds for the canonical shifts.

Let $R$ be the von Neumann algebra generated by the standard tower obtained from $A \supset \Gamma(A)$. Since $\Gamma$ is a basic *-endomorphism of $A$, there exists a *automorphism of $R$ which is an extension of $\Gamma$. We denote it by $\Theta$. 
Theorem 26. Let $N \subset M$ be type $\mathrm{II}_{1}$-factor with finite index. Then the automorphism $\Theta$ induced by the canonical shift $\Gamma$ for the inclusion $N \subset M$ is ergodic. If $N \subset M$ has finite depth

$$
H(M \mid N)=H(\Theta)=H(\Gamma)=\log [M: N] .
$$

Proof. Let us take an $x \in R$ such that $\Theta(x)=x$. By considering the standard tunnel obtained through $\Gamma$,

$$
\cdots \subset N_{k}=M_{-k} \subset \cdots \subset N_{1}=N=M_{-1} \subset M_{0}=M \subset M_{1} \subset \cdots \subset M_{j} \subset \cdots
$$

we observe that $R$ is generated by $\bigcup_{k, j}\left(N_{k}^{\prime} \cap M_{j}\right)$. Then for any $\varepsilon>0$ there are $k$ and $j$ such that $\left\|x-x^{\prime}\right\|_{2}<\varepsilon$ for some $x^{\prime} \in N_{k}^{\prime} \cap M_{j}$. Since $\Theta$ is trace preserving, $\left\|x^{\prime}-\Theta\left(x^{\prime}\right)\right\|_{2}<2 \varepsilon$. On the other hand $\Theta^{m}\left(x^{\prime}\right) \in N_{k-2 m}^{\prime} \cap M_{j+2 m}$ for all $m$ and $\left(N_{k-2 m}^{\prime} \cap M_{j+2 m}\right) \cap\left(N_{k}^{\prime} \cap M_{j}\right)=\mathbf{C} 1$ for a large enough $m$. Hence $x \in \mathbf{C 1}$. Assume that $N \subset M$ has finite depth. Then $\Theta$ is a 2-shift on the tower $\left(M_{-k}^{\prime} \cap M_{j}\right)_{k, j}$ for $R$ by the same proof as one for $\Gamma$. Since $M_{-k}^{\prime} \cap M_{j}$ is isomorphic to $A_{j+k}$, we have by Theorem 1,

$$
H(\Theta)=\lim _{k \rightarrow \infty} \frac{H\left(M_{-k}^{\prime} \cap M_{k}\right)}{k}=\lim _{k} \frac{H\left(M^{\prime} \cap M_{2 k}\right)}{k}=H(\Gamma) .
$$

Hence we have the relation by Corollary 24 .

\section{ACKNOWLEDGMENTS}

The author would like to express her thanks for the hospitality of the organizers U. Haagerup and E. Størmer at the Mittag-Leffler Institute and also A. Connes and M. Takesaki at the Institute des Hautes Etudes Scientifiques during the spring of 1989.

\section{REFERENCES}

1. M. Choda, Shifts on the hyperfinite $\mathrm{II}_{1}$-factor, J. Operator Theory 17 (1987), 223-235.

2. _ـ Entropy for *-endomorphisms and relative entropy for subalgebras, J. Operator Theory (to appear).

3. A. Connes and E. Størmer, Entropy for automorphism of $\mathrm{II}_{1}$ von Neumann algebras, Acta Math. 134 (1975), 288-306.

4. A Connes, H. Narnhofer, and W. Thirring, Dynamical entropy of $C^{*}$-algebras and von Neumann algebras, Comm. Math. Phys. 112 (1987), 691-719.

5. F. Goodman, P. de la Harpe, and V. Jones, Coxeter graphs and towers of algebras, Math. Sci. Res. Inst. Publ. 14, Springer-Verlag, 1989.

6. V. F. R. Jones, Index for subfactors, Invent. Math. 72 (1983), 1-25.

7. R. Longo, Simple injective subfactors, Adv. in Math. 63 (1987), 152-171.

8. - Solution of the factorial Stone-Weierstrass conjecture. An application of the theory of standard split $W^{*}$-inclusions, Invent. Math. 76 (1984), 145-155.

9. A. Ocneanu, Quantized groups, string algebras, and Galois theory for algebras, Preprint.

10. M. Pimsner and S. Popa, Entropy and index for subfactors, Ann. Sci. École Norm. Sup. 19 (1986), 57-106.

11. __ Iterating the basic construction, Trans. Amer. Math. Soc. 310 (1988), 127-134.

12. S. Popa, Maximal injective subalgebras in factors associated with free groups, Adv. in Math. 50 (1983), 27-48.

13. __ Classification of subfactors: The reduction to commuting squares, Preprint. 
14. R. T. Powers, An index theory for semigroups of ${ }^{*}$-endomorphisms of $B(H)$ and type $\mathrm{II}_{1}$ factors, Canad. J. Math. 40 (1988), 86-114.

15. M. Takesaki, Theory of operator algebras. I, Springer-Verlag, 1979.

16. H. Umegaki, Conditional expectations in an operator algebra, Tôhoku Math. J. 6 (1954), 358-362.

17. H. Wenzl, Representation of Hecke algebras and subfactors, Thesis, Univ. of Pennsylvania, 1985.

Department of Mathematics, Osaka Kyoiku University, Tennoji, Osaka 543 Japan

E-mail address: f00527@sinet.ad.jp 